\title{
Strain Differences in Intraluminal Thread Model of Middle Cerebral Artery Occlusion in Rats
}

\author{
J. SVOBODA ${ }^{1 *}$, A. LITVINEC ${ }^{1 *}$, D. KALA ${ }^{1}$, A. POŠUSTA $^{1}$, L. VÁVROVÁ $^{1}$, P. JIRUŠKA $^{1}$, \\ J. OTÁHAL ${ }^{1}$ \\ * These authors contributed equally to this work. \\ ${ }^{1}$ Institute of Physiology of the Czech Academy of Sciences, Prague, Czech Republic
}

Received June 7, 2018

Accepted August 9, 2018

Epub Ahead of Print October 23, 2018

\section{Summary}

Stroke is despite of progressive improvements in treatment and reperfusion strategies one of the most devastating human pathology. However, as quality of acute health care improves and more people survive ischemic attack, healthcare specialists have to solve new challenges to preserve reasonable quality of life to these patients. Thus, novel approaches which prevents comorbidities of stroke and improve quality of life of stroke survivors in general has to be developed and experimentally tested. The aim of the present paper was to establish reliable rat model of middle cerebral occlusion and set of methods allowing selection of animals suitable for long-term experiments. We have compared mortality rates, cerebral blood flow and extension of ischemic lesion induced by intraluminal filament in three widely used outbred rat strains. We have additionally used an animal 18F-DG PET scans to verify its reliability in noninvasive detection of ischemic infarct in acute period ( $24 \mathrm{~h}$ after MCAO) for selecting animals eligible for long survival experiments. Our data clearly indicates that high variability between rat strains might negatively influence stroke induction by intraluminal thread occlusion of middle cerebral artery. Most reliable outbred rat strain in our hands was Sprague-Dawley where maximal reduction of cerebral blood flow and extensive ischemic lesion was observed. Contrary, Wistar rats exhibited higher mortality and Long-Evans rats significantly smaller or no ischemic region in comparison to Sprague-Dawley. Additionally, we have confirmed a positron emission tomography with $18 \mathrm{~F}$-fluorodeoxyglucose as suitable method to assess extension of ischemic region in acute period after the experimental arterial occlusion in rats.

\section{Key words}

Focal cerebral ischemia • Middle cerebral artery • Occlusion • Intraluminal Thread $\bullet$ Rat

\section{Corresponding author}

J. Otáhal, Institute of Physiology of the Czech Academy of Sciences, Vídeňská 1083, 14220 Prague 4, Czech Republic. E-mail: jakub.otahal@fgu.cas.cz

\section{Introduction}

Despite progressive improvements in novel treatment and reperfusion strategies stroke remains one of the most devastating human pathology. Mortality highly depends on the health care system and life style and thus significantly geographically varies; however, even in countries with highly accessible and good quality health care stroke is second leading cause of death (Thrift et al. 2017). According to the WHO statistics, more than 15 million people suffer stroke worldwide annually (HEALTH IN 2015: FROM MDGs TO SDGs, available from http://www.who.int/gho/publications/mdgssdgs/en/). Up to 5 million patients (one third) die to the acute stroke and another 5 million are permanently disabled. Although risk of stroke increases with age a significant proportion of strokes occur in productive age and thus represents substantial social and economic burden for affected persons and their families but also to the society (Daniel et al. 2009). Beside adult age a perinatal period represents high risk to child and perinatal stroke and/or hypoxia-ischemia still affect 
relevant portion of both term and preterm birth babies causing whole life handicap or death (Brima et al. 2013a, Brima et al. 2013b, Riljak et al. 2016). The cause of more than $80 \%$ of strokes is occlusion of the cerebral vessel (ischemic stroke) which results in energy and oxygen deprivation and accumulation of metabolic waste. As a consequence, a myriad of processes including excitotoxic action of glutamate, oxidative stress, calcium dependent degradation and other lead to irreversible injury of the brain tissue. The only causal and effective treatment strategy is early restoration of the blood flow. Brain tissue surrounding ischemic core (penumbra) maintains partial level of blood supply by vessel anastomosis can be rescued when blood supply is reestablished within critical time window (Dirnagl et al. 1999). In present, therapeutic window extends up to $4.5 \mathrm{~h}$ for fibrinolytic treatment and $6 \mathrm{~h}$ for mechanical recanalization strategy after the occurrence of the first signs of the stroke (Powers et al. 2015) although recent studies suggests that some patients may profit from reperfusion even later after this time window (Cheripelli et al. 2016). Historically, beside reperfusion therapy (the only causal treatment of stroke) several attempts have been made to pharmacologically extend the limited time window for reperfusion therapy. Neuroprotective strategies including NMDA blockers or free radical scavengers have been successfully tested in experimental models but with no success in clinical trials. For example a lazaroid, tirilazade mesylate, an inhibitor of lipid peroxidation, has been withdrawn from clinical testing after exhibiting no or even worsening effect (Cahill and Hall 2017). Failure of translation of knowledge from preclinical animal research to human clinics emerges limitations of experimental approaches. Therefore, guidelines have been widely discussed and published to improve outcomes of preclinical stroke research (Liu et al. 2009, Albers et al. 2011).

In present, an extensive accessibility of reperfusion therapies in developed countries significantly improved survival rate in acute stroke and thus brings novel challenges to preclinical research in neurology. Individuals suffering stroke might develop severe long-term consequences including vascular epilepsy, which considerably aggravate the quality of their lives. Therefore, there is a need for reliable experimental model mimicking both acute and chronic consequences of human stroke. Reliability of the animal model of stroke highly depends on technique used to occlude blood vessel and various factors including animal strain and age of animals used (Albers et al. 2011). During past decades various techniques of occlusion of brain vessels were developed. Direct surgical occlusion of MCA (Tamura et al. 1981), endovascular occlusion with intraluminal filament (Longa et al. 1989), photothrombic model (Brima et al. 2013a, Brima et al. 2013b) and several types of embolic occlusion using various embolizing elements (autologous blood clot, glass microspheres, magnetic nanoparticles, for review see (Fluri et al. 2015)) mimic important patterns of human stroke. However, none of these models is ideal for all purposes and thus optimal model should be selected to get appropriate answer to the particular experimental question. In our present paper, we have established intraluminal filament occlusion model based on Longa's method (Longa et al. 1989) and compared several parameters of stroke between three widely used outbred rat strains during acute period of stroke. We have additionally used an animal PET scanner to verify its reliability in detection of ischemic infarct in acute period ( $24 \mathrm{~h}$ after MCAO) for selecting animals eligible for long survival experiments.

\section{Methods}

In total 34 adult male rats (280-320 g) were used for these experiments. We have used three different outbred rat strains - Wistar, Long Evans (both from local breeding based on Charles River strain) and SpragueDawley (obtained from Charles River). The protocol of experiments was approved by the Animal Care and Use Committee of the Institute of Physiology, Academy of Sciences of the Czech Republic, in agreement with Animal Protection Law of the Czech Republic, which is fully compatible with the guidelines of the European Community Council directives 86/609/EEC. The Institute possesses The Statement of Compliance with Standards of Humane Care and Use of Laboratory Animals \#A5228-01 from NIH. All efforts were made to minimize animal suffering and to reduce the number of animals used.

Animals were anesthetized in inducing chamber using 3-4\% of Isoflurane. Spontaneously breathing animals were weighted and transferred to a heating surgical pad, anesthesia maintained with $1-2 \%$ of Isoflurane and rectal temperature were measured and controlled to maintain at $37.1 \pm 0.25^{\circ} \mathrm{C}$. To monitor effect of middle cerebral occlusion on blood flow a laser Doppler flowmeter probe was fixed to the parietal bone over the MCA territory by means of cyanoacrylate glue. The middle cerebral artery occlusion (MCAO) was 
performed according to slightly modified procedure originally described by Longa et al. (1989). Briefly, after preparation of surgical field a sagittal neck incision was made to approach carotic triangle. Under surgical microscope a bifurcation of common carotid artery (CCA) and outgoing trunks of internal (ICA) and external carotid (ECA) arteries with its branches were carefully exposed from surrounding soft tissues. A vascular microclip was placed over CCA and ICA. ECA was ligated over the origin of thyroid artery and occipital artery electrocoagulated and cut to prevent retrograde blood flow. From small arteriotomy an appropriate nylon filament with silicon tip (Doccol, USA) was inserted into ECA and carefully advanced into an origin of ICA and after slight ligation and microclip removal further advanced into the ICA (Fig. 1). The movement of the filament was visually inspected to prevent misplacement of the filament into a pterygopalatine artery. After small resistance was felt and simultaneous significant drop in CBF detected by LDF the filament was secured by tightening of the ligature. Animals were left for desired period of occlusion $(60 \mathrm{~min}$.) under mild anesthesia (0.5-1.2\% Isoflurane) and temperature control. Then occluding filament was carefully withdrawn from the artery and arteriotomy closed with ligation to prevent further bleeding. Finally, microvascular clip was removed from CCA and surgical field closed with three to four sutures. Postsurgical period was covered with nonsteroid antiflogistic Meloxicam ( $2 \mathrm{mg} / \mathrm{kg}$ s.c.). Animals were routinely checked and weighted during postsurgical recovery to assess their overall status and motor deficit using Garcia score (Garcia et al. 1995).

Cerebral blood flow was monitored and recorded during entire surgical procedure using surface microprobe (Probe 407-1, Perimed, for placement see above) connected to laser Doppler flowmeter (LDF, Periflow 5010, Primed, Sweden). The analog LDF signal was connected to 16bit A/D converter Power 1401 and recorded using Spike2 software (CED, Cambridge, United Kingdom). Data were analyzed offline in Spike2 software to assess mean level of the regional cerebral blood ( $\mathrm{rCBF}$ ) flow during desired periods of experiments.

To evaluate extension of ischemic region animals were sacrificed $24 \mathrm{~h}$ after MCAO and their brains quickly removed from the skull. Fresh coronal brain slices were cut using rat brain metal matrix with blade. Tetrazolium (2,3,5-Triphenyl-2H-tetrazolium chloride) staining was performed according to commonly used protocol (Liszczak et al. 1984). Briefly, one-millimeter thick fresh brain slices were incubated in $2 \%$ tetrazolium at room temperature for 25-30 min. Images of stained slices were then captured using standard color camera and stored for further quantitative analysis.

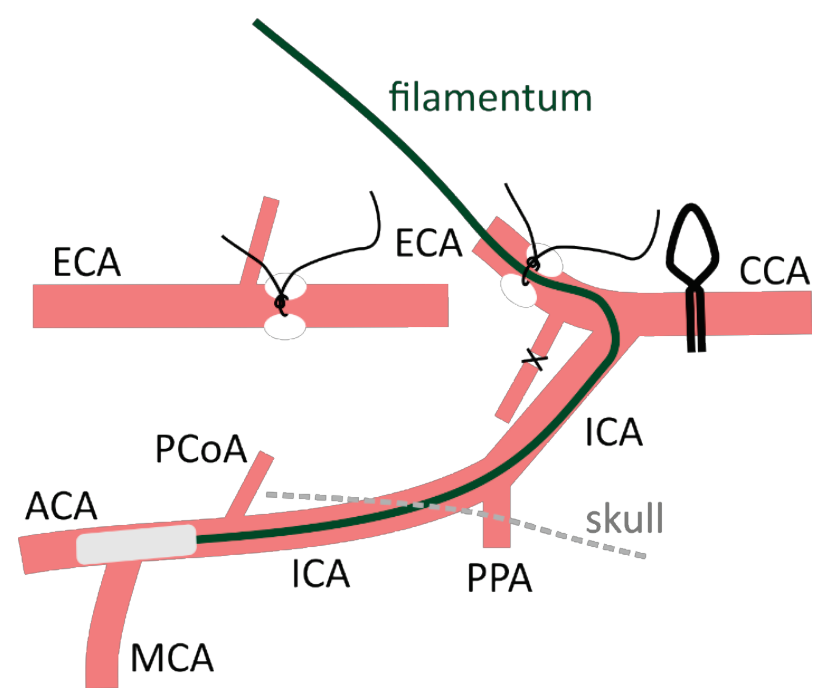

Fig. 1. Schematic drawing of surgical procedure of intraluminal occlusion of middle cerebral artery. An intraluminal filament with silicon tip is introduced through internal carotid artery (ICA) into the circle of Willis to occlude middle cerebral artery (MCA). ECA external carotid artery, CCA - common carotid artery, ACA anterior cerebral artery, PCOA - posterior communicating artery, PPA - pterygopalatine artery.

In 6 animals (Sprague-Dawley) a series of $\mu \mathrm{CT} / \mathrm{PET}$ scans was performed to verify its suitability for noninvasive assessment of ischemic region in intraluminal MCAO. First series of $\mu \mathrm{CT} / \mathrm{PET}$ were captured $24 \mathrm{~h}$ before and second $24 \mathrm{~h}$ after the MCAO procedure. The acquisition was performed according to adapted protocol of Balsara et al. (2014). Briefly, animals were fasted overnight and $1 \mathrm{~h}$ before $\mu \mathrm{CT} / \mathrm{PET}$ scan were injected with $\sim 18 \mathrm{MBq}$ of $18 \mathrm{~F}$-FDG diluted in saline to total volume $0.4 \mathrm{ml}$. The head of the animal were scanned in the Albira animal scanner (Albira, Bruker, USA) for 30 min followed by CT scan in standard resolution. The data were analyzed offline in PMOD software (PMOD technologies LLC, Zurich, Switzerland) with use of Schiffers MRI rat brain atlas which implements the Paxinos coordinates allowing to assess FDG activity bilaterally in majority of brain regions (Schiffer et al. 2006). 3D visualizations of the animal including brain with ischemic lesion were constructed from NIfTI data format of both $\mu \mathrm{CT}$ and PET utilizing 3D Slicer software (www.slicer.org, NIH, USA).

Data were statistically compared using paired t-test or ANOVA for repeated measures or ANOVA on 
Ranks where appropriate. Data are expressed as mean \pm SEM. Level of statistical significance was set to $\mathrm{P}<0.05$ (*).

\section{Results}

Surgical procedure was comparable between the three rat strains included in the study and no obvious difference in vascular anatomy of the neck vessels was observed. Only 2 animals out of 34 died (5.9\%) within $4 \mathrm{~h}$ after reperfusion period. Interestingly, both animals have been from Wistar rat strain. After physical inspection of the brain and the skull occurrence of subarachnoid hemorrhage was likely the cause of death. Temperature had tendency to slightly increase $\left(+0.3^{\circ} \mathrm{C}\right)$ after occlusion of MCA (data not shown). We have observed delicate weight reduction during $24 \mathrm{~h}$ period after the MCAO in all three animal strains while only Wistar rats exhibited significant reduction of $20.7 \pm 4.6 \mathrm{~g}$ ( $\mathrm{P}<0.05$, Fig. 2). All animals behave well after the MCAO and exhibit no or moderate neurological deficit (data not shown).

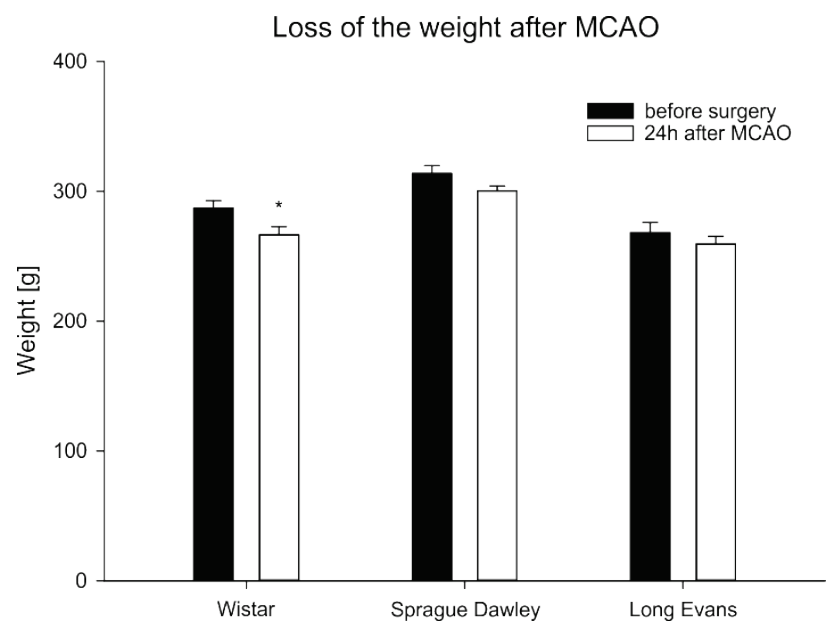

Fig. 2. Surgical procedure and occlusion of middle cerebral artery led to decrease of weight of the animals.

Intraluminal occlusion of MCA origin resulted in ischemic injury to the affected hemisphere in all animals. Most extensive area of infarct, expressed as ratio of area of tetrazolium positive lesion to area of hemisphere, has been observed in Sprague-Dawley $(50.2 \pm 10.3 \%)$ and Wistar rats $(36.8 \pm 5.6 \%)$ while LongEvans exhibited significantly smaller region of cerebral ischemia $(18.8 \pm 3.5 \%, \mathrm{P}<0.05)$. Similar pattern has been observed when rostro-caudal extension of the ischemic lesion has been compared (Fig. 3). The affected areas differ between the rat strains. In Sprague-Dawley and Wistar animals where the infarcts were most extensive, the intraluminal occlusion of middle cerebral artery typically induced ischemic lesion affecting basal ganglia (caudoputamen), piriform and entorhinal cortices, primary somatosensory and marginally motor cortex. In contrast, only moderate infarcts of caudoputamen with delicate extension to surrounding sensorimotor cortex have been observed in Long-Evans animals $24 \mathrm{~h}$ after the MCAO (Fig. 4).
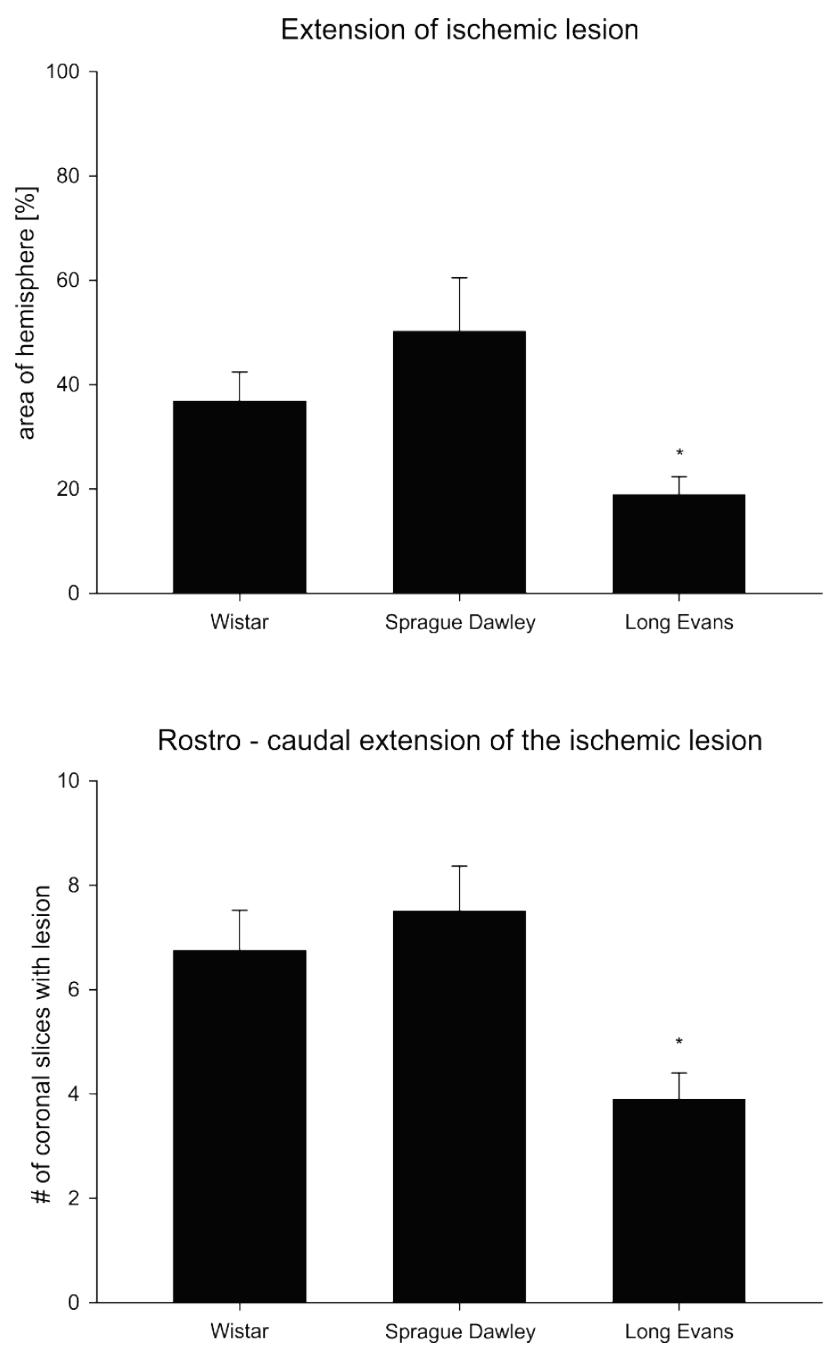

Fig. 3. Extension of the ischemic lesion was significantly higher in Sprague-Dawley and Wistar rats in comparison to Long-Evans rats.

Regional cerebral blood flow tightly follows steps of the surgical procedure affecting cerebral blood inflow, which can be seen on typical LDF traces in Figure 5a. Basal regional cerebral blood flow did not significantly differ between strains and exhibited physiological oscillations arising from cardiac and ventilation activity. First significant drop in rCBF 
occurred with placement of microvascular clip on CCA. This value of $\mathrm{rCBF}$ was later used as reference resting level of the blood flow. Introduction of the filament tip into the MCA origin produced sudden decrease of $\mathrm{rCBF}$ in all rat strains namely to $48.0 \pm 6.2 \%$ In Wistar, to $37.5 \pm 6.0 \%$ in Sprague-Dawley and to $63.2 \pm 5.9$ in LongEvans rats. During period of occlusion (60 $\mathrm{min}$ ) the rCBF had tendency to gradually but non-significantly increase. Withdrawal of the occluding filament from its position after the occluding period led to steep increase of the rCBF typically to values detected during insertion of the filament just before occluding MCA or even little higher $(98.0 \pm 5.7 \%$ in Wistar, $112.2 \pm 19.9 \%$ in Sprague-Dawley and $97.2 \pm 8.4 \%$ in Long-Evans rats). The release of the clip from CCA caused additional increase of the $\mathrm{rCBF}$ as response to reperfusion of carotid territory (Wistar 131.1 \pm 13.0 , Sprague-Dawley 143.2 \pm 26.0 , Long-Evans $140.0 \pm 14.8 \%$; Fig. 5b).

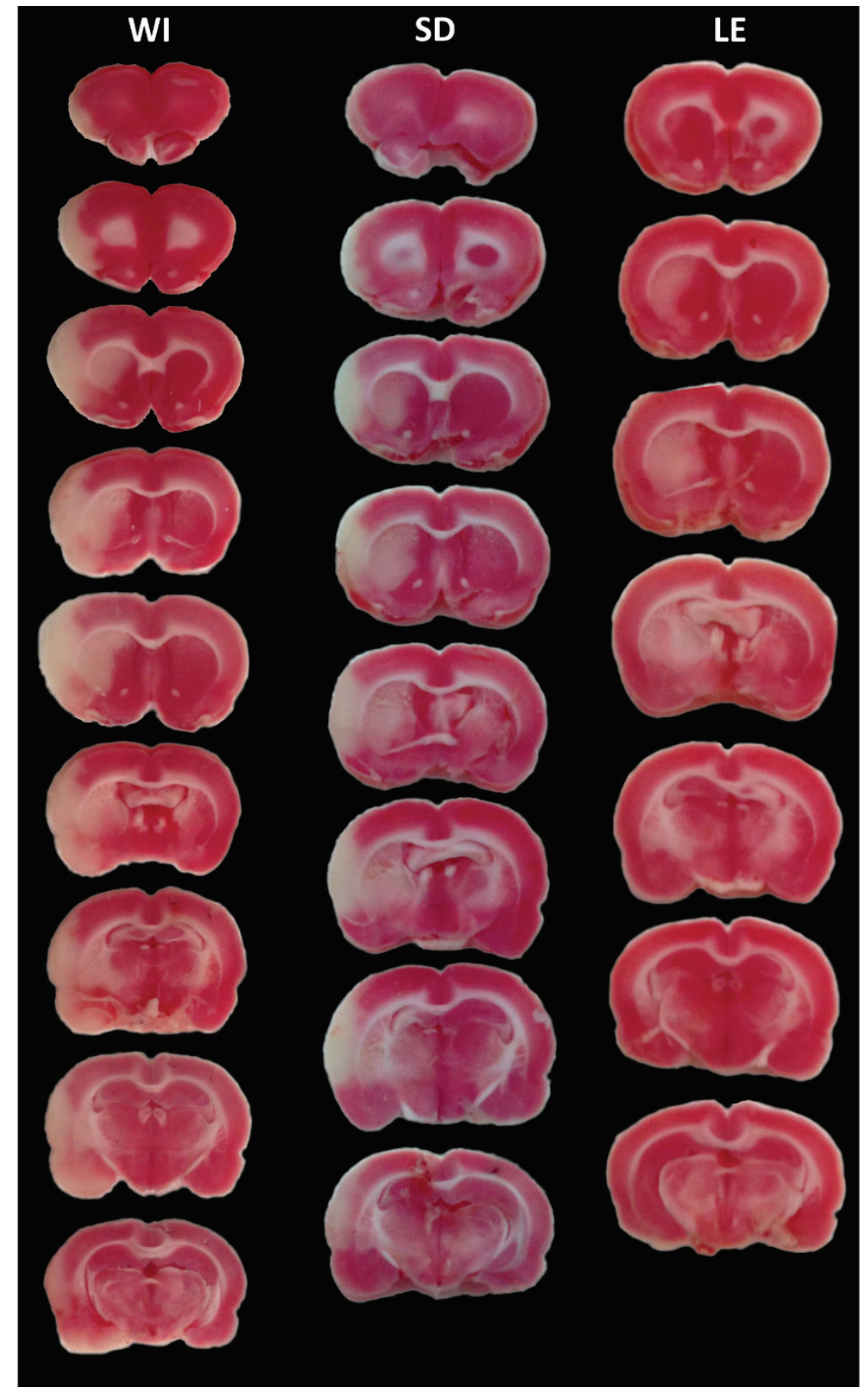

Fig. 4. Tetrazolium staining revealed ischemic region of the brain (loss of pink color) in all three rat strains (WI - Wistar, SD - SpragueDawley, LE - Long-Evans). 
A

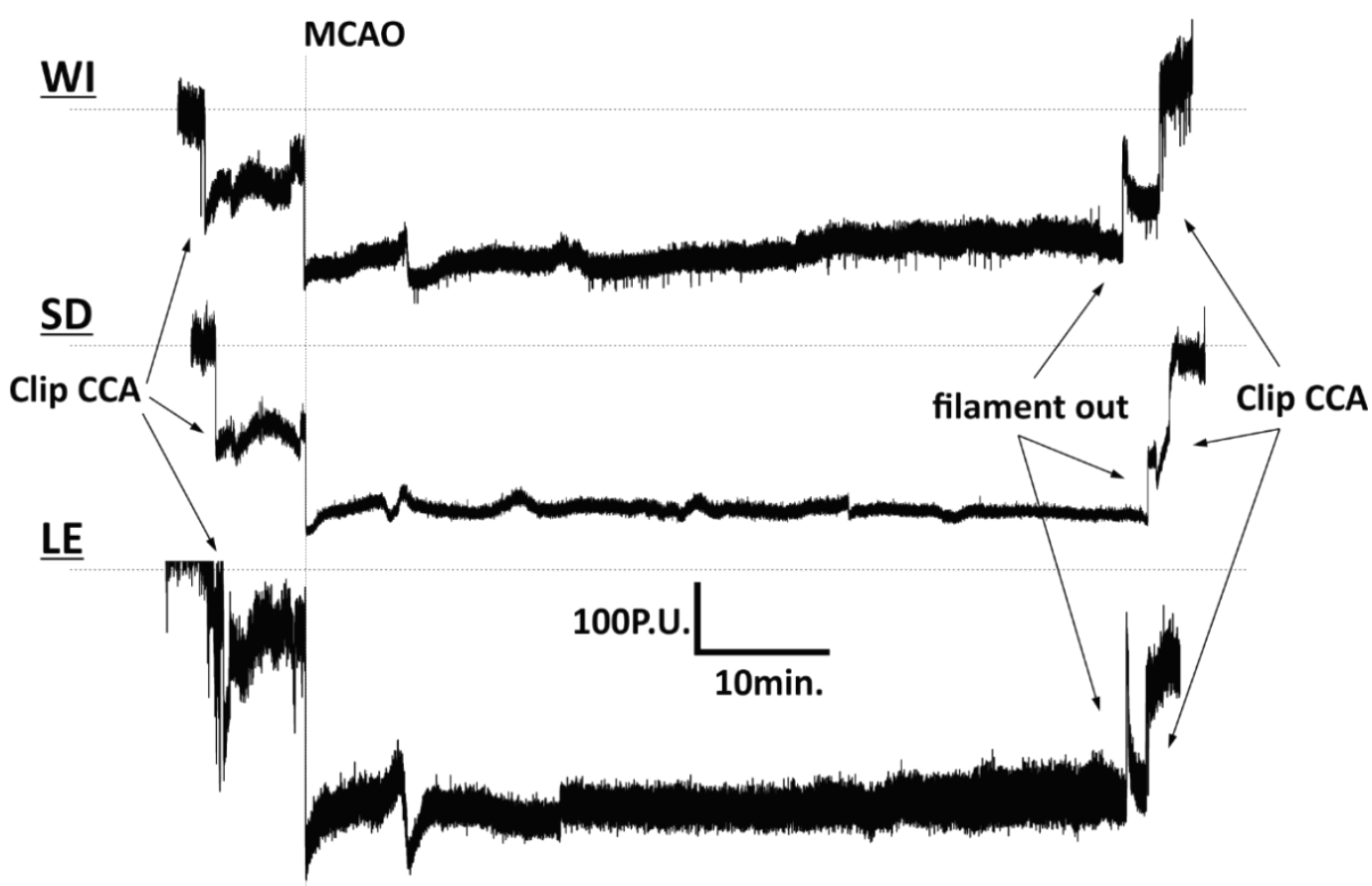

B

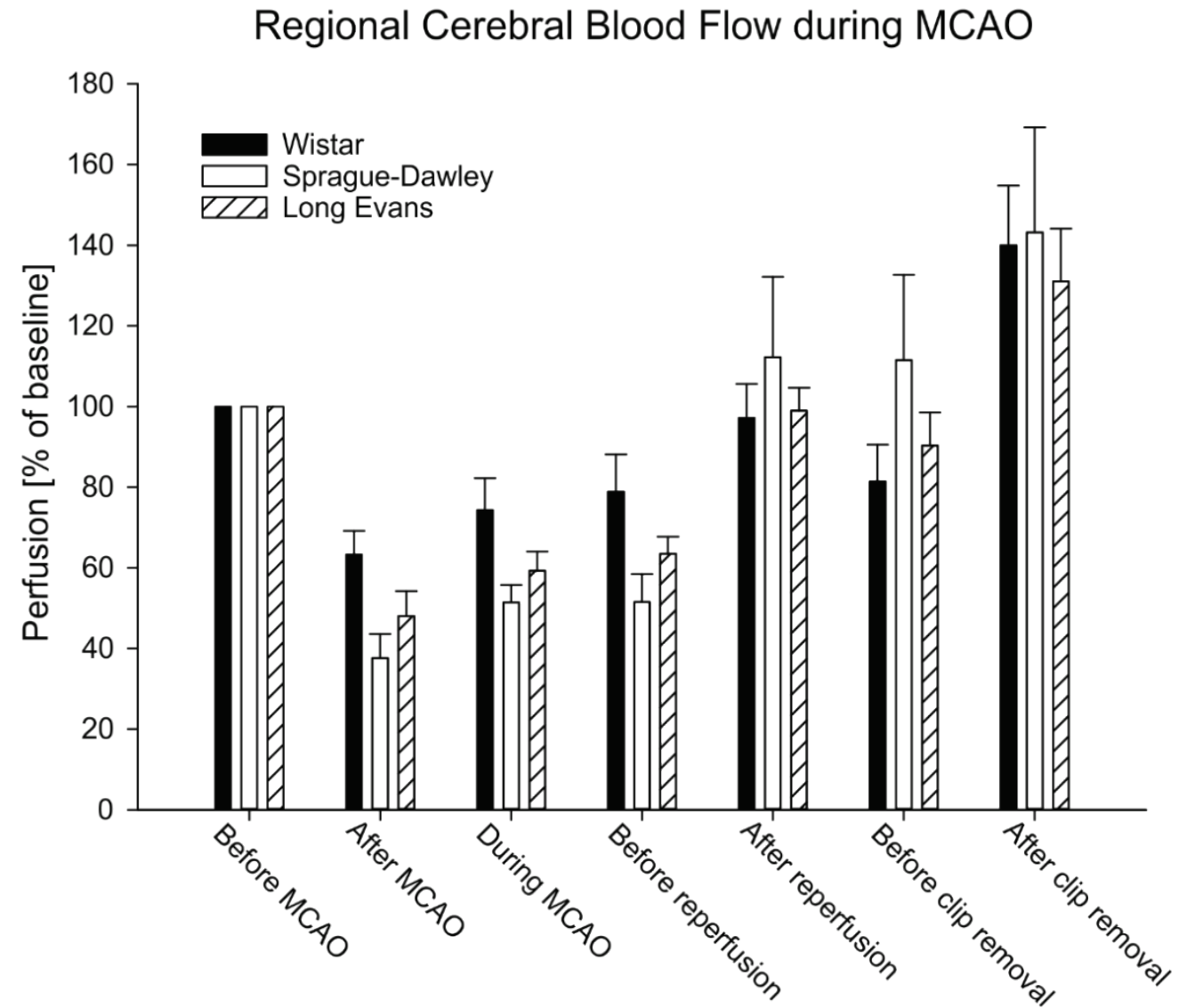

Fig. 5. Regional cerebral blood flow was significantly reduced after the occlusion of middle cerebral artery (MCAO). After the removal of occluding filament and vascular microclip the cerebral blood flow returns to pre-occlusion level. (A) shows representative measurement of CBF with LDF. $*$ P $<0.05$ when compared to initial value (Before MCAO). (B) Shows statistics of cerebral blood flow in selected regions of interest throughout the surgery in between the strains. 
Cerebral ischemia produced significant reduction in focal brain metabolism as revealed by 18F-FDG PET scan. Representative PET images and 3D reconstruction are present in Figure 6. Cerebral ischemia was clearly visible as large hypometabolic region anatomically corresponding to ischemic region as revealed with tetrazolium staining of the same animal. Quantification of 18F-FDG activity in several brain regions were done using MRI atlas after space anatomical registration to PET and CT image series (for details see Methods). Additionally, ratios between right and left side were calculated for individual neuroanatomical structures segmented by MRI atlas in both hemispheres (Fig. 7). A noticeable increase of the $\mathrm{R} / \mathrm{L}$ index of the FDG activity was observed in all regions corresponding to the ischemic regions as revealed by tetrazolium staining (caudoputamen, amygdala, hypothalamus, diencephalon and several neocortical regions) while only cingulate exhibit statistically significant difference when comparing activities before and after MCAO. This was likely caused by small number of animals which underwent PET scan, however, obvious difference in signal from all other ischemic regions allowed us visually delineate ischemic region for every individual animal.
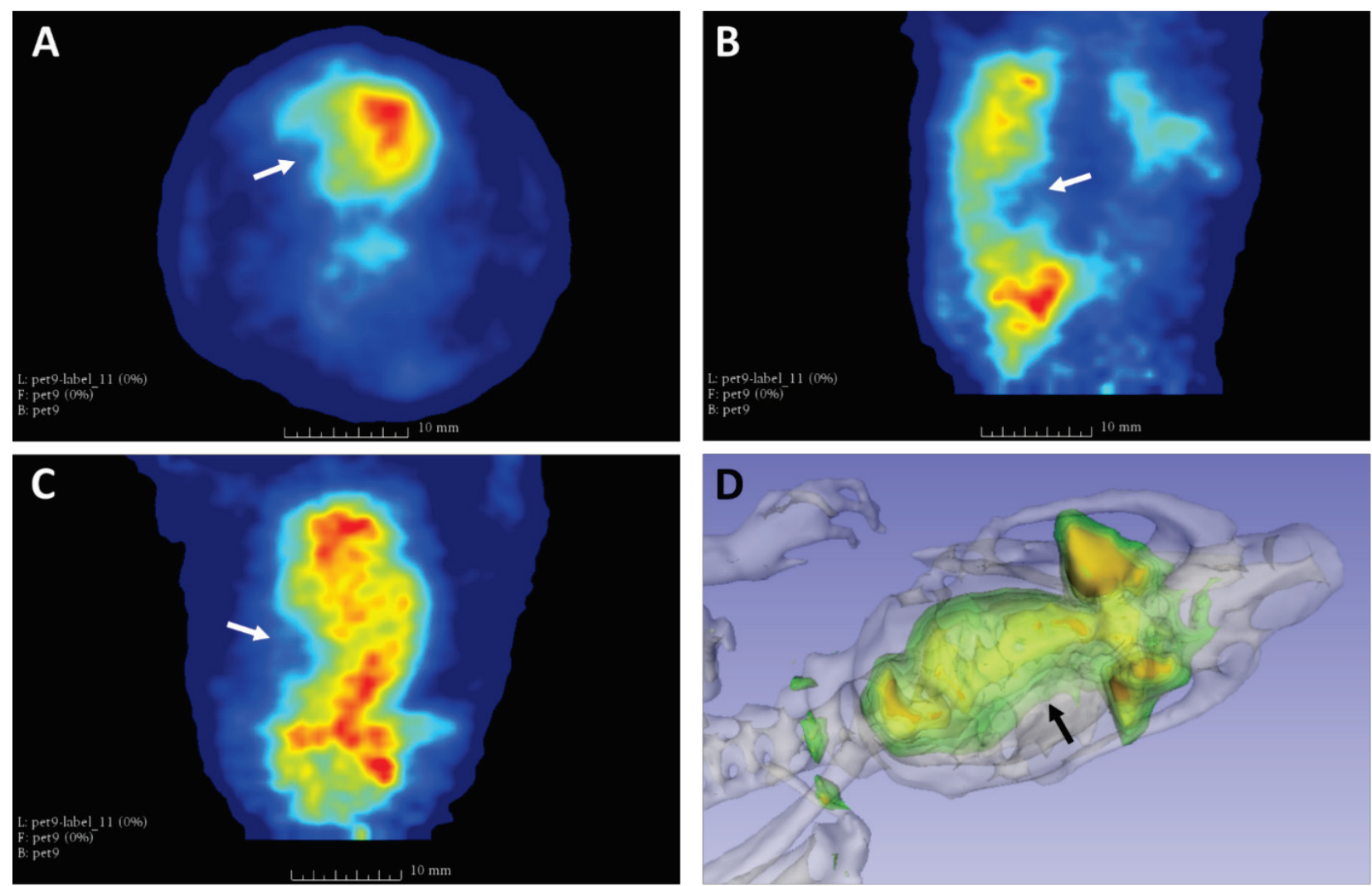

$\mathbf{E}$

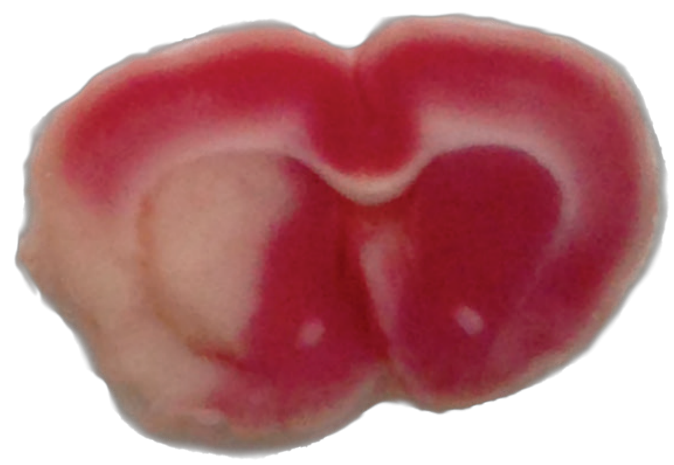

Fig. 6. 18F-fluorodeoxyglucose PET scans $24 \mathrm{~h}$ after MCAO and reperfusion in Sprague-Dawley rats revealed extensive hypometabolic focus corresponding to ischemic region, which has been visualized postmortem by tetrazolium staining. (A-D) 18F-fluorodeoxyglucose PET scans $24 \mathrm{~h}$ after MCAO and reperfusion in Sprague-Dawley rats revealed extensive hypometabolic focus corresponding to ischemic region. (E) Visualization of ischemic region in the same rat by tetrazolium staining. 


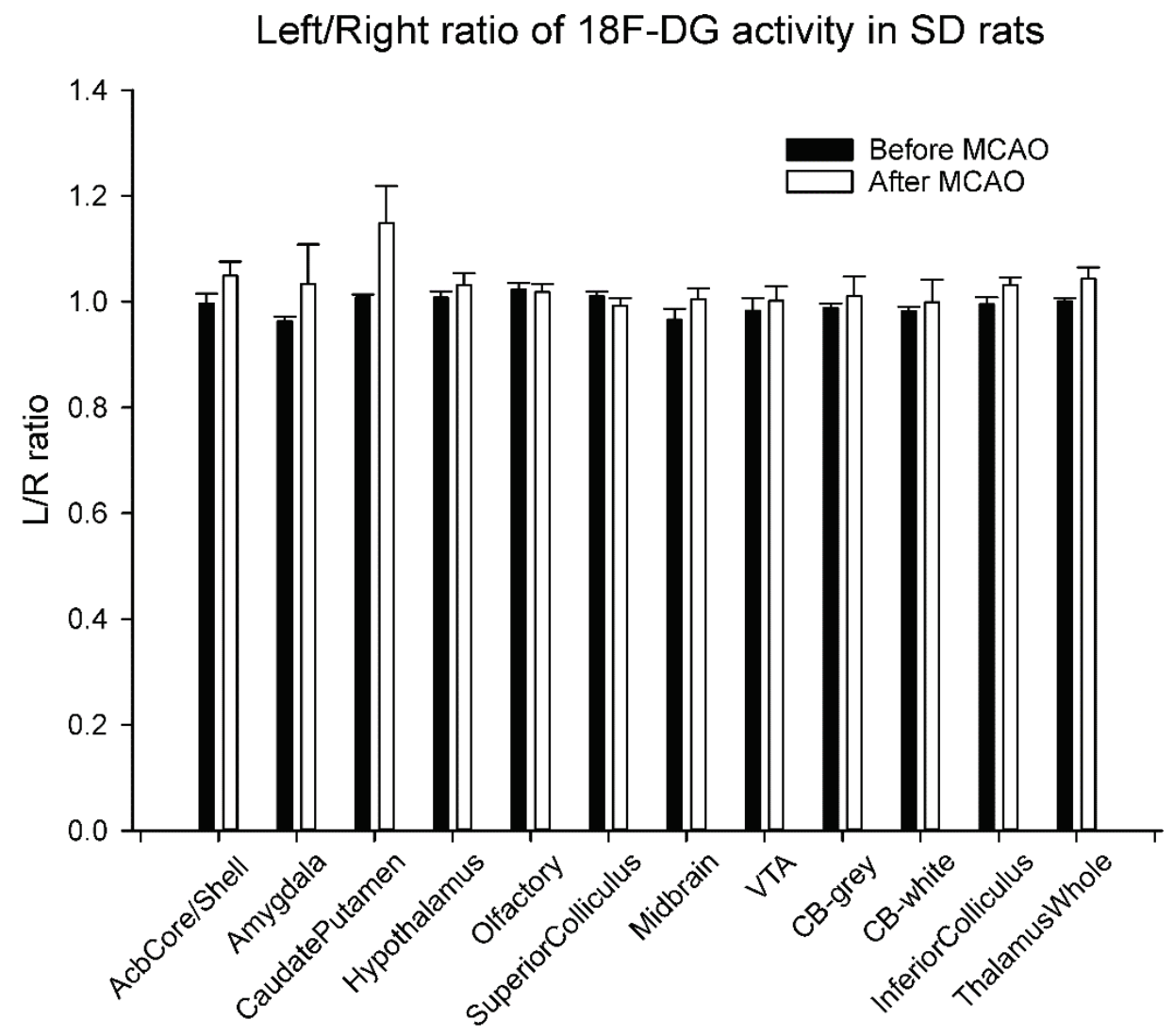

Left/Right ratio of 18F-DG activity in SD rats

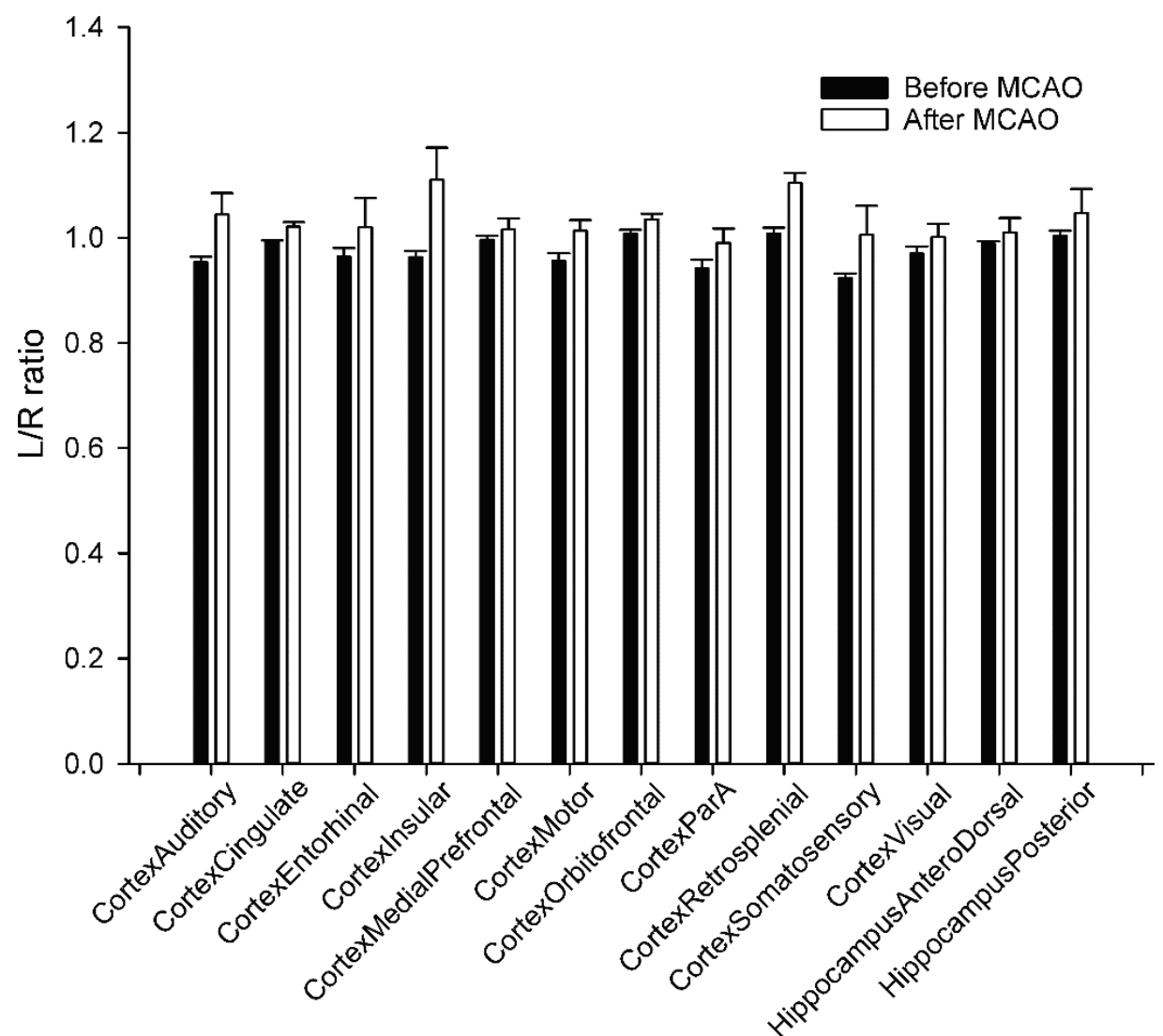

Fig. 7. Statistical evaluation of $18 \mathrm{~F}$-fluorodeoxyglucose activity in selected brain regions expressed as ratio left/right hemisphere. 


\section{Discussion}

We have shown that extension of ischemic region significantly differs between animal strains. We have compared Wistar, Long-Evans and Sprague-Dawley rat strains. Although brain-supplying vessels macroscopically do not differ in between the strains our results of regional cerebral blood flow during occlusion suggest distinct meso and microvasculature of the brain. Cerebral blood flow dropped down to one third of preocclussion levels in Sprague-Dawley. Contrary, in Wistar rats or Long-Evans the less severe decrease of $\mathrm{rCBF}$ was observed, to half in Wistar and to two thirds in Long-Evans rats respectively. Although ischemic region was reliably detected on tetrazolium staining in all three strains most consistent results have been observed in Sprague-Dawley rats. Reason why occlusion of the origin of middle cerebral artery results in different decline in $\mathrm{rCBF}$ is likely due to a distinct net of collateral anastomoses. It has been shown previously anatomy of terminal brain vessels significantly differs between rat strains and also between different vendors (Oliff et al. 1997). Another explanation of inter-strain difference observed might be a various sensitivity to ischemic insults due to delicate variances in brain metabolism. Indeed, it has been shown recently that levels of excitatory neurotransmitters naturally differs and thus it might explain for example higher susceptibility to seizures in strains having higher resting levels of glutamate (Fauvelle et al. 2015). We can hypothesize that also inter-strain or even inter-individual differences in brain metabolism might alter sensitivity to ischemic insult. However, till now there is limited evidence in the literature. Altogether, in our conditions adult Sprague-Dawley outbred rats obtained from Charles River exhibited most reliable results.

Cerebral blood flow is typically monitored during whole period of occluding procedure. For this purpose, a laser Doppler flowmetry is typically used. The sensing probe is fixed to thinned skull allowing to monitor cerebral perfusion in surrounding brain tissue. According to the principle of the method, the value is provided in arbitrary units (P.U. - perfusion units) which does not represent quantitative measure of blood flow per volume or weight unit of nervous tissue. This method thus allows comparative evaluation of blood flow typically before and after the occlusion. The critical step of the procedure is correct placement and fixation of LDF probe to prevent any movements. Continuous monitoring of $\mathrm{rCBF}$ with LDF allowed us to detect sudden reduction of $\mathrm{rCBF}$ which occurred during occlusion as well as fast increase in consequence of withdrawal of occluding filament. In two animals which died during or early after the surgical procedure a slow increase of $\mathrm{rCBF}$ after filament withdrawal was observed suggesting subarachnoid hemorrhage (Woitzik and Schilling 2002) which was consequently proven as presence of massive blood deposition on the skull basis during autopsy. The fact that both animals which died during procedure were Wistar rats further limit usability of this strain in intraluminal MCAO. Reperfusion led to increase of the $\mathrm{rCBF}$, which was even significantly higher in comparison to basal levels. This is likely caused by release of vasoactive substances e.g. nitric oxide which results in brain vasodilatation (Iadecola and Nedergaard 2007, Brozickova and Otahal 2013).

Occlusion of middle cerebral artery with intraluminal filament resulted in generation of extensive ischemic region as revealed by tetrazolium staining $24 \mathrm{~h}$ after the reperfusion. We decided to test 60 min occlusion interval because this time interval is on the lower edge of time window when reperfusion therapy might be appropriately provided to patients. Thus, it is likely that longer or permanent occlusion will lead to larger infarcts and presumably also to smaller differences between rat strains. Extension of ischemic lesion well correspond to levels of cerebral blood flow measured after the occlusion of MCA. Most extensive lesion was detected in SpragueDawley affecting almost half of the brain hemisphere including cerebral cortex and subcortical structures like basal ganglia. Contrary, Long-Evans rats developed less severe ischemia extending to fifth of the brain hemisphere dominantly affecting subcortical structures namely caudoputamen. The subcortical extension of ischemic lesion also explains less severe drop in cerebral blood flow, because cortical blood flow is mainly detected when surface LDF probe is used (Taninishi et al. 2015). Obtained results in Sprague-Dawley and Wistar rats were comparable to those published elsewhere (Longa et al. 1989, Bardutzky et al. 2005).

Massive use of reperfusion strategies in clinical practice as well as substantial shortening of the times from stroke initiation to reperfusion almost reaching limits of healthcare open new challenges for preclinical research. Increasing numbers of people successfully surviving stroke might largely benefit from secondary prevention and strategies improving their quality of life. Rehabilitation and compensatory aids help people to 
recover their motor functions or compensate their motor deficits. However, patients suffering stroke are commonly in risk of severe conditions which my negatively alter their quality of life after stroke. Epilepsy, depression and anxiety are some of them and thus it is important to perform long-term poststroke experiments with aim to understand mechanism responsible for their development. To limit number of experimental animals in groups it is thus important to select those with similar extension of lesion directly after the stroke induction. Therefore we have tested whether 18F-DG PET scanning $24 \mathrm{~h}$ after the stroke induction is capable to reliably detect and evaluate ischemic region. According to results obtained from tetrazolium staining we have selected Sprague-Dawley outbred rat strain for further testing. In agreement with Balsara (2014) our results clearly indicates that PET can be used for outlining ischemic brain region $24 \mathrm{~h}$ after reperfusion. Ischemic region has been detected as region with decreased $18 \mathrm{~F}$ activity in comparison to remaining brain tissue. 18F-DG utilize same transport mechanisms as glucose and thus when intravenously injected it is distributed into metabolically active tissue according to activity of glucose transporters. When 18F-DG transported into cells it is converted with first enzyme of glucose metabolism and is not further capable to neither enter consequent steps of glycolysis nor leave the cell. 18F-DG thus accumulates in metabolically active cells. Observed hypometabolism thus represent decrease in cerebral glucose metabolism in affected tissue. We have utilized standard evaluation of 18F-DG activity based on left/right comparison and visual inspection. However, it has been shown recently that by employing more advanced mathematical analysis of the PET data, namely Statistical Parametric Mapping, it is possible to highly improve detection capabilities of the method (Nie et al. 2014). Additionally, recent preclinical studies suggest that more specific radiotracers detecting neuroinflammation can be used after the stroke to predict occurrence of spontaneous recurrent seizures later during life (Bertoglio et al. 2017). Functional imaging by means of positron emission tomography seems to be promising method, which can elucidate processes leading to development of pathologies, which typically accompany stroke recovery such epilepsy or depression and help to discover strategies to prevent them.

In conclusion, our data clearly indicates that high variability between rat strains might negatively influence stroke induction by intraluminal thread occlusion of middle cerebral artery. Most reliable outbred rat strain in our hands was Sprague-Dawley. Contrary, Wistar rats exhibited higher mortality and Long-Evans rats significantly smaller or no ischemic region in comparison to Sprague-Dawley. Additionally, we have confirmed a positron emission tomography with $18 \mathrm{~F}$-fluorodeoxyglucose as suitable method to assess extension of ischemic region in acute period after the experimental arterial occlusion in rats.

\section{Conflict of Interest}

There is no conflict of interest.

\section{Acknowledgements}

The study was supported by grant project number 1533115A from the Czech Health Research Council. The core facility used in this project is supported by MEYS project „LM2015062 Czech-BioImaging“ and by project ERDF, OPPK „BIOMODELS CZ.2.16/3.1.00/24017“.

\section{References}

ALBERS GW, GOLDSTEIN LB, HESS DC, WECHSLER LR, FURIE KL, GORELICK PB, HURN P, LIEBESKIND DS, NOGUEIRA RG, SAVER JL, CONSORTIUM SV: Stroke Treatment Academic Industry Roundtable (STAIR) recommendations for maximizing the use of intravenous thrombolytics and expanding treatment options with intra-arterial and neuroprotective therapies. Stroke 42: 2645-2650, 2011.

BALSARA RD, CHAPMAN SE, SANDER IM, DONAHUE DL, LIEPERT L, CASTELLINO FJ, LEEVY WM: Non-invasive imaging and analysis of cerebral ischemia in living rats using positron emission tomography with 18F-FDG. $J$ Vis Exp 94: 51495, 2014.

BARDUTZKY J, SHEN Q, HENNINGER N, BOULEY J, DUONG TQ, FISHER M: Differences in ischemic lesion evolution in different rat strains using diffusion and perfusion imaging. Stroke 36: 2000-2005, 2005.

BERTOGLIO D, VERHAEGHE J, SANTERMANS E, AMHAOUL H, JONCKERS E, WYFFELS L, VAN DER LINDEN A, HENS N, STAELENS S, DEDEURWAERDERE S: Non-invasive PET imaging of brain inflammation at disease onset predicts spontaneous recurrent seizures and reflects comorbidities. Brain Behav Immun 61: 69-79, 2017. 
BRIMA T, MIKULECKA A, OTAHAL J: Impacts of perinatal induced photothrombotic stroke on sensorimotor performance in adult rats. Physiol Res 62: 85-94, 2013a.

BRIMA T, OTAHAL J, MARES P: Increased susceptibility to pentetrazol-induced seizures in developing rats after cortical photothrombotic ischemic stroke at P7. Brain Res 1507: 146-153, $2013 \mathrm{~b}$.

BROZICKOVA C, OTAHAL J: Effect of an inhibitor of neuronal nitric oxide synthase 7-nitroindazole on cerebral hemodynamic response and brain excitability in urethane-anesthetized rats. Physiol Res 62 (Suppl 1): S57-S66, 2013.

CAHILL L, HALL ED: Is it time to resurrect "lazaroids"? J Neurosci Res 95: 17-20, 2017.

DANIEL K, WOLFE CD, BUSCH MA, MCKEVITT C: What are the social consequences of stroke for working-aged adults? A systematic review. Stroke 40: e431-e440, 2009.

DIRNAGL U, IADECOLA C, MOSKOWITZ MA: Pathobiology of ischaemic stroke: an integrated view. Trends Neurosci 22: 391-397, 1999.

FAUVELLE F, BOCCARD J, CAVAREC F, DEPAULIS A, DERANSART C: Assessing susceptibility to epilepsy in three rat strains using brain metabolic profiling based on HRMAS NMR spectroscopy and chemometrics. J Proteome Res 14: 2177-2189, 2015.

FLURI F, SCHUHMANN MK, KLEINSCHNITZ C: Animal models of ischemic stroke and their application in clinical research. Drug Des Devel Ther 9: 3445-3454, 2015.

GARCIA JH, WAGNER S, LIU KF, HU XJ: Neurological deficit and extent of neuronal necrosis attributable to middle cerebral artery occlusion in rats. Statistical validation. Stroke 26: 627-634; discussion 635, 1995.

CHERIPELLI BK, HUANG X, MCVERRY F, MUIR KW: What is the relationship among penumbra volume, collaterals, and time since onset in the first $6 \mathrm{~h}$ after acute ischemic stroke? Int $J$ Stroke 11: 338-346, 2016.

IADECOLA C, NEDERGAARD M: Glial regulation of the cerebral microvasculature. Nat Neurosci 10: 1369-1376, 2007.

LISZCZAK TM, HEDLEY-WHYTE ET, ADAMS JF, HAN DH, KOLLURI VS, VACANTI FX, HEROS RC, ZERVAS NT: Limitations of tetrazolium salts in delineating infarcted brain. Acta Neuropathol 65: 150-157, 1984.

LIU S, ZHEN G, MELONI BP, CAMPBELL K, WINN HR: Rodent stroke model guidelines for preclinical stroke trials (1st edition). J Exp Stroke Transl Med 2: 2-27, 2009.

LONGA EZ, WEINSTEIN PR, CARLSON S, CUMMINS R: Reversible middle cerebral artery occlusion without craniectomy in rats. Stroke 20: 84-91, 1989.

NIE B, LIU H, CHEN K, JIANG X, SHAN B: A statistical parametric mapping toolbox used for voxel-wise analysis of FDG-PET images of rat brain. PLoS One 9: e108295, 2014.

OLIFF HS, COYLE P, WEBER E: Rat strain and vendor differences in collateral anastomoses. J Cereb Blood Flow Metab 17: 571-576, 1997.

POWERS WJ, DERDEYN CP, BILLER J, COFFEY CS, HOH BL, JAUCH EC, JOHNSTON KC, JOHNSTON SC, KHALESSI AA, KIDWELL CS, MESCHIA JF, OVBIAGELE B, YAVAGAL DR, AMERICAN HEART ASSOCIATION STROKE C: 2015 American Heart Association/American Stroke Association Focused Update of the 2013 Guidelines for the Early Management of Patients With Acute Ischemic Stroke Regarding Endovascular Treatment: A Guideline for Healthcare Professionals From the American Heart Association/American Stroke Association. Stroke 46: 3020-3035, 2015.

RILJAK V, KRAF J, DARYANANI A, JIRUSKA P, OTAHAL J: Pathophysiology of perinatal hypoxic-ischemic encephalopathy - biomarkers, animal models and treatment perspectives. Physiol Res 65 (Suppl 5): S533-S545, 2016.

SCHIFFER WK, MIRRIONE MM, BIEGON A, ALEXOFF DL, PATEL V, DEWEY SL: Serial microPET measures of the metabolic reaction to a microdialysis probe implant. J Neurosci Methods 155: 272-284, 2006.

TAMURA A, GRAHAM DI, MCCULLOCH J, TEASDALE GM: Focal cerebral ischaemia in the rat: 1. Description of technique and early neuropathological consequences following middle cerebral artery occlusion. J Cereb Blood Flow Metab 1: 53-60, 1981. 
TANINISHI H, JUNG JY, IZUTSU M, WANG Z, SHENG H, WARNER DS: A blinded randomized assessment of laser Doppler flowmetry efficacy in standardizing outcome from intraluminal filament MCAO in the rat. J Neurosci Methods 241: 111-120, 2015.

THRIFT AG, THAYABARANATHAN T, HOWARD G, HOWARD VJ, ROTHWELL PM, FEIGIN VL, NORRVING B, DONNAN GA, CADILHAC DA: Global stroke statistics. Int J Stroke 12: 13-32, 2017.

WOITZIK J, SCHILLING L: Control of completeness and immediate detection of bleeding by a single laser-Doppler flow probe during intravascular middle cerebral artery occlusion in rats. J Neurosci Methods 122: 75-78, 2002. 